\title{
Visual information without thermal energy may induce thermoregulatory-like cardiovascular responses
}

Jun'ya Takakura ${ }^{*}$, Takayuki Nishimura ${ }^{2}$ and Shigeki Watanuki ${ }^{3}$

\begin{abstract}
Background: Human core body temperature is kept quasi-constant regardless of varying thermal environments. It is well known that physiological thermoregulatory systems are under the control of central and peripheral sensory organs that are sensitive to thermal energy. If these systems wrongly respond to non-thermal stimuli, it may disturb human homeostasis.

Methods: Fifteen participants viewed video images evoking hot or cold impressions in a thermally constant environment. Cardiovascular indices were recorded during the experiments. Correlations between the 'hot-cold' impression scores and cardiovascular indices were calculated.

Results: The changes of heart rate, cardiac output, and total peripheral resistance were significantly correlated with the 'hot-cold' impression scores, and the tendencies were similar to those in actual thermal environments corresponding to the impressions.

Conclusions: The present results suggest that visual information without any thermal energy can affect physiological thermoregulatory systems at least superficially. To avoid such 'virtual' environments disturbing human homeostasis, further study and more attention are needed.
\end{abstract}

Keywords: Video, Visual information, Impressions, Cardiovascular responses, Physiological thermoregulatory systems, Virtual reality

\section{Background}

Maintaining a quasi-constant core body temperature is essential for sustaining human life. This physiological temperature regulation system can be roughly divided into two parts comprising sensors and effectors. Sensors have sensitivity to thermal energy (temperature), and are distributed throughout the central and peripheral body areas. Sensory thermal information is integrated in the preoptic area of the hypothalamus, which is thought to be the central component of thermoregulation [1]. According to this sensory information, the hypothalamus then drives effectors. For example, in a situation where the body temperature should be lowered, vasodilation and increased cardiac output occur to facilitate heat radiation. In contrast, in

\footnotetext{
* Correspondence: fridge_lab@kyudai.jp

${ }^{1}$ Graduate School of Integrated Frontier Science, Kyushu University, 4-9-1,

Shiobaru, Minami-Ku, Fukuoka, Japan

Full list of author information is available at the end of the article
}

a situation where body temperature should be elevated, vasocontraction and decreased cardiac output occur to hinder heat radiation [2-4].

This human thermoregulatory system is often compared to manmade systems [5], such as an air conditioner, which also has sensors and effectors that work to maintain a constant air temperature. These systems work because engineers select the appropriate sensors (for example, a thermistor, which senses temperature) to detect thermal energy. If inappropriate sensors (for example, a phototransistor, which senses visible light) are selected and embedded in the system, temperature regulation would be disturbed [6], as visible light has almost no thermal energy.

Unlike manmade systems, however, the human thermoregulatory system developed over the course of evolution rather than being designed by rational engineers [7]. Thus, inappropriate sensors may have been incorporated into

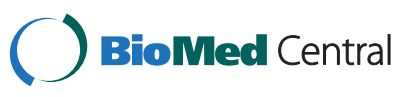


the human thermoregulatory system. Although a wholly inadequate design would not have been selected in the course of evolution, a design that is imperfect but did not have destructive consequences in the context of evolution may have been able to persist [8]. Assuming that thermal stimuli are always accompanied by non-thermal stimuli, then sensitivity to non-thermal stimuli is synonymous with sensitivity to thermal stimuli, because it can act as actual physiological thermal sensors instead. Thermoregulatory systems that are partly under the control of non-thermal stimuli work well as long as both thermal stimuli and non-thermal stimuli correlate with each other, in which case such a system would not have been maladaptive in the course of human evolution. For example, visual information can act as non-thermal stimuli; for example, presumably, when human ancestors were exposed to a cold environment, visual stimuli such as snow or ice were also apparent.

In today's environment, media technologies have enabled us to be exposed to visual stimuli corresponding to hot or cold temperatures independent of the actual thermal environment. In other words, thermal stimuli and non-thermal stimuli have begun to dissociate, and if non-thermal stimuli can exert significant effects on thermal regulation, this may have negative consequences. Considering the rapid increase in the use of electronic media [9], the effects of non-thermal stimuli on homeostasis should be considered. The purpose of this study was to examine whether visual information that for human ancestors would have been accompanied by actual thermal stimuli (that is, hot or cold environments) affects human thermoregulatory physiological responses in the absence of any actual thermal stimulus.

\section{Methods}

Fifteen male graduate and undergraduate students (age, 20 to 24 years old) participated in this study. The experimental protocol consisted of a total of nine sessions. The time course of each session is shown in Figure 1. After the sensors were attached to the participants, they sat on a chair in front of a display (PFM-42B1, Sony, Tokyo, Japan). After 5 minutes of viewing a gray screen, videos were presented for 10 minutes. After viewing the images for 8 minutes, participants rated their subjective impressions of the images and their thermal sensations on a 7-point scale from -3 (very cold) to +3 (very hot). Impressions of the images on a 7-point scale about pleasantness (like-dislike) were also recorded. Here, 'impressions' refer to participants' subjective feelings about the videos and 'thermal sensations' refer to their actual physical sensations of temperature. The participants' drowsiness was checked between the experimental sessions and they were allowed to talk with the experimenter to keep the arousal level. The presented images comprised sceneries of icebergs (FILM1), snow (FILM2), drifting ice (FILM3), a tropical forest (FILM4), a desert (FILM5), an erupting volcano (FILM6), a stream (FILM7), autumn leaves (FILM8), and a gray screen (FILM9). Examples of the images are shown in Figure 2. The nine images were presented in random order to avoid ordering effects. The temperature of the experimental room was set at $28^{\circ} \mathrm{C}$ and the humidity was set at $50 \%$ of relative humidity. The experiment was conducted in autumn in Japan.

Physiological signals were measured continuously throughout the sessions. Electrocardiography (ECG) electrodes were attached to the chest and amplified by an amplifier (AB-621G, Nihon Kohden, Tokyo, Japan). Impedance cardiography was measured using a tape-electrode and an impedance meter (AI-601G, Nihon Kohden), and its time derivation was also calculated (ED-601G, Nihon Kohden). Continuous blood pressure was measured through the finger (Finapress 2300, Ohmeda, Amsterdam, Netherlands). These analog signals were digitalized by an $\mathrm{A} / \mathrm{D}$ converter at a sampling rate of $1,000 \mathrm{~Hz}$ and stored on a personal

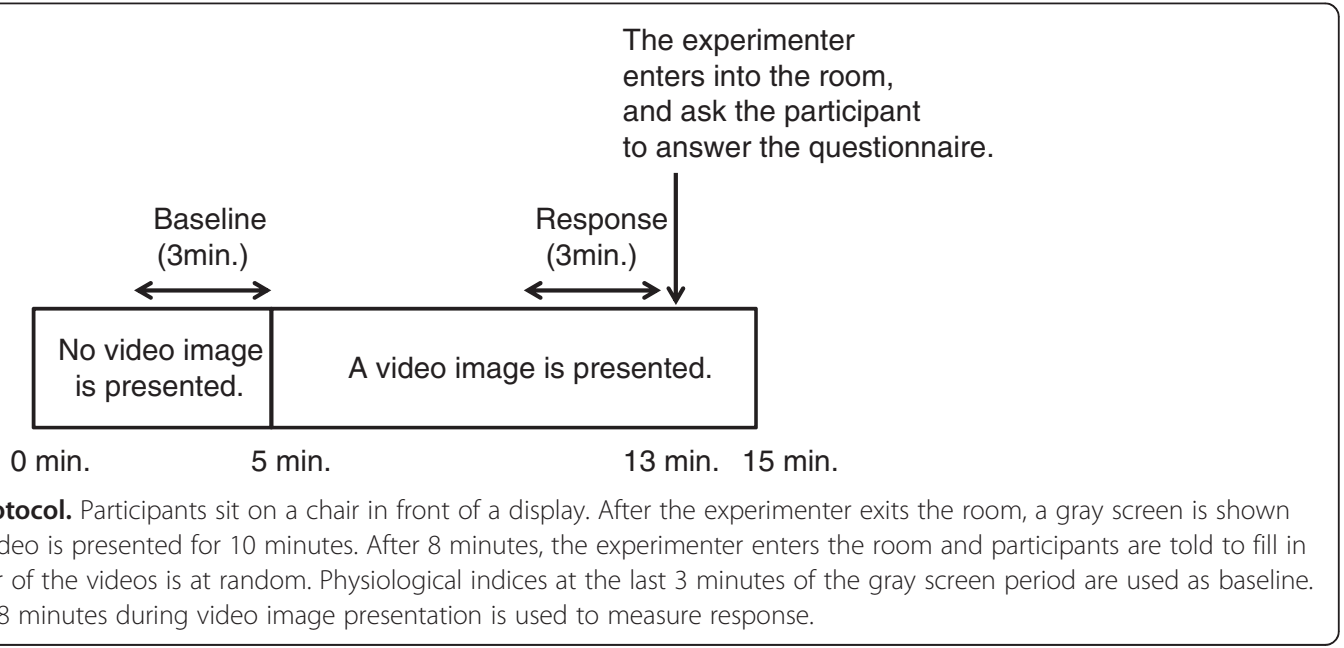




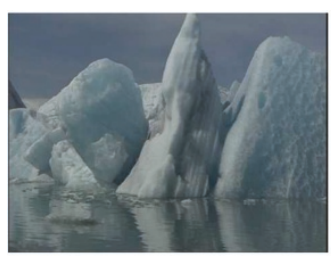

FILM1

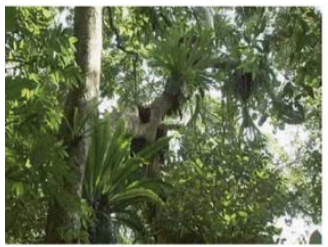

FILM4

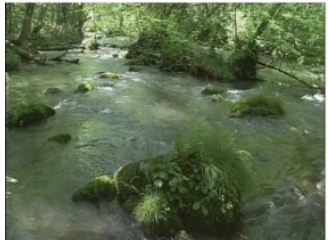

FILM7

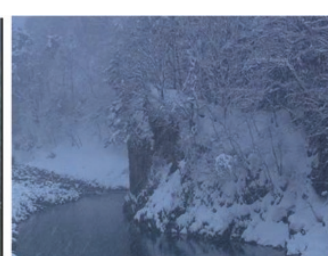

FILM2

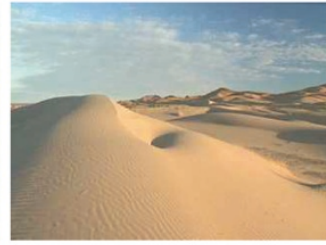

FILM5

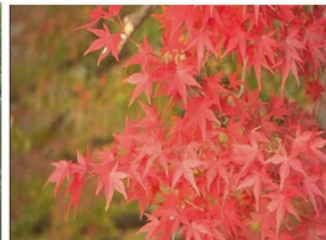

FILM8

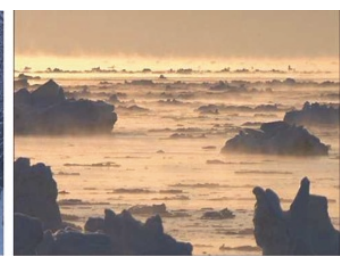

FILM3

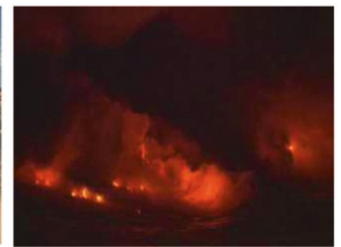

FILM6

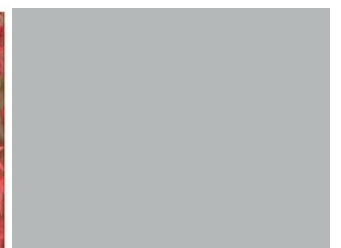

FILM9

Figure 2 Examples of videos. There were nine videos (FILM1 to FILM9) recorded on DVD. The contents of each are: FILM1, icebergs; FILM2, snow; FILM3, drifting ice; FILM4, tropical forest; FILM5, desert; FILM6, erupting volcano; FILM7, stream; FILM8, autumn leaves; and FILM9, gray screen.

computer. The experimental procedure was approved by the local ethics committee of the Faculty of Design, Kyushu University (Fukuoka, Japan). All participants provided written informed consent.

Offline analysis was conducted using in-house software developed in our laboratory. Cardiovascular indices were calculated beat-by-beat. Heart rate was derived from the R waves of ECG. Stroke volume was calculated using Kubicek's formula [10] by derivation from impedance cardiography, which was filtered by an adaptive filter [11]. Systolic and diastolic blood pressures were the upper and lower peaks of the blood pressure waveform. Mean blood pressure was calculated by averaging the waveform. Cardiac output and total peripheral resistance were also calculated based on the above indices. Heart rate variability (HRV) was calculated using the maximum entropy method and the power spectrum was divided into three components: $\mathrm{HF}(0.15 \mathrm{~Hz}$ to $0.40 \mathrm{~Hz}), \mathrm{LF}(0.06 \mathrm{~Hz}$ to $0.15 \mathrm{~Hz}$ ), and VLF (0.02 Hz to $0.06 \mathrm{~Hz})$.

Changes from baseline were calculated for each index: heart rate $(\Delta \mathrm{HR})$, stroke volume $(\Delta \mathrm{SV})$, cardiac output $(\triangle \mathrm{CO})$, systolic blood pressure $(\triangle \mathrm{SBP})$, diastolic blood pressure $(\triangle \mathrm{DBP})$, mean blood pressure $(\triangle \mathrm{MBP})$, total peripheral resistance $(\triangle \mathrm{TPR})$, high frequency $\mathrm{HRV}(\triangle \mathrm{HF})$, low frequency $\mathrm{HRV}(\triangle \mathrm{LF})$, and very low frequency of $\operatorname{HRV}(\triangle \mathrm{VLF})$.

To confirm that each video invoked a different impression, we applied the Friedman test to the 'hot-cold' impression score. We did not conduct a post-hoc test, because this was not our main purpose.

We used multiple regression analysis to determine the relationship between cardiovascular indices and impression scores among the participants [12]. We used cardiovascular indices as dependent variables, and impression scores and participants as independent variables. Participants were treated as categorical factors using dummy variables with 14 degrees of freedom. The $P$ value from the t-test for the regression slope (the common coefficient $a$ described as below) of impression scores was used to determine the probability of the analysis. The magnitude of the intra-individual correlation coefficients between the impression score and the cardiovascular indices among the participants was calculated as:

$|r|=\sqrt{\frac{\text { sum of squares for impression score }}{\text { sum of squares for impression score }+ \text { residual sum of square }}}$

The sign of the correlation coefficients is given by that of the regression coefficients for impression score. Note that the above $r$ is not the multiple regression correlation coefficient and the $P$ value is not the result of overall multiple regression analysis. Although this method is useful for many purposes, many readers may not be familiar with this method. Thus, we explain this method somewhat minutely. 
We collected both independent variables (impression scores) and dependent variables (cardiovascular indices) for each participant. The data for each participant comprised several points. If we take the data in Figure 3A, then this sample consists of five participants and each participant has five data points. Applying correlation analysis to these data, there is a positive correlation between independent variables and dependent variables, as shown in Figure 3B. However, looking at the data intraindividually, a negative correlation is seen, as shown in Figure 3C. Multiple regression analysis using dummy variables identified an intra-individual correlation. Here, we establish $y(i, j)$ as the dependent variable and $x(i, j)$ as the independent variable, where $i$ denotes the participant $(i=\mathrm{A}, \mathrm{B}, \mathrm{C}, \mathrm{D}$, and $\mathrm{E})$ and $j$ is the intra-individual data indicator $(j=1,2, \ldots, 5)$. In addition, we insert dummy independent variables $x d_{\mathbf{k}}(i, j)(k=\mathrm{B}, \mathrm{C}, \mathrm{D}$, and E), defined as:

$$
x d_{k}(i, j)=\left\{\begin{array}{cc}
1 & i=k \\
0 & i \neq k
\end{array}\right.
$$

Now we have one-dimensional dependent variables $y(i, j)$ and five-dimensional independent variables consisting of $x(i, j)$ and $x d_{\mathrm{k}}(i, j)$. Applying multiple regression analysis to these data, we obtain the following regression formula:

$$
\begin{aligned}
\hat{y}(i, j)= & a x(i, j)+a_{B} x d_{B}(i, j)+a_{C} x d_{C}(i, j) \\
& +a_{D} x d_{D}(i, j)+a_{E} x d_{E}(i, j)+b
\end{aligned}
$$

In the above formula, $a$ is the common coefficient that explains the intra-individual slopes. Each $a_{k}$ is biased for each participant. If the common coefficient $a$ is non-zero, it means that the dependent variable $y(i, j)$ and the independent variable $x(i, j)$ are correlated intra-individually and that tendency is common among participants (see Additional file 1). Figure 3D is a schematic explanation of the regression formula.

There are possible confounding factors that affect cardiovascular responses other than hot or cold visual information. If the participants feel 'like' or 'dislike' about the video images, it can also induce cardiovascular responses. In addition, although we set the room air temperature constant, it is unavoidable to fluctuate in some degree, and possibly induce actual thermoregulatory cardiovascular responses. To confirm these possibilities, we conducted the same regression analysis adapting the
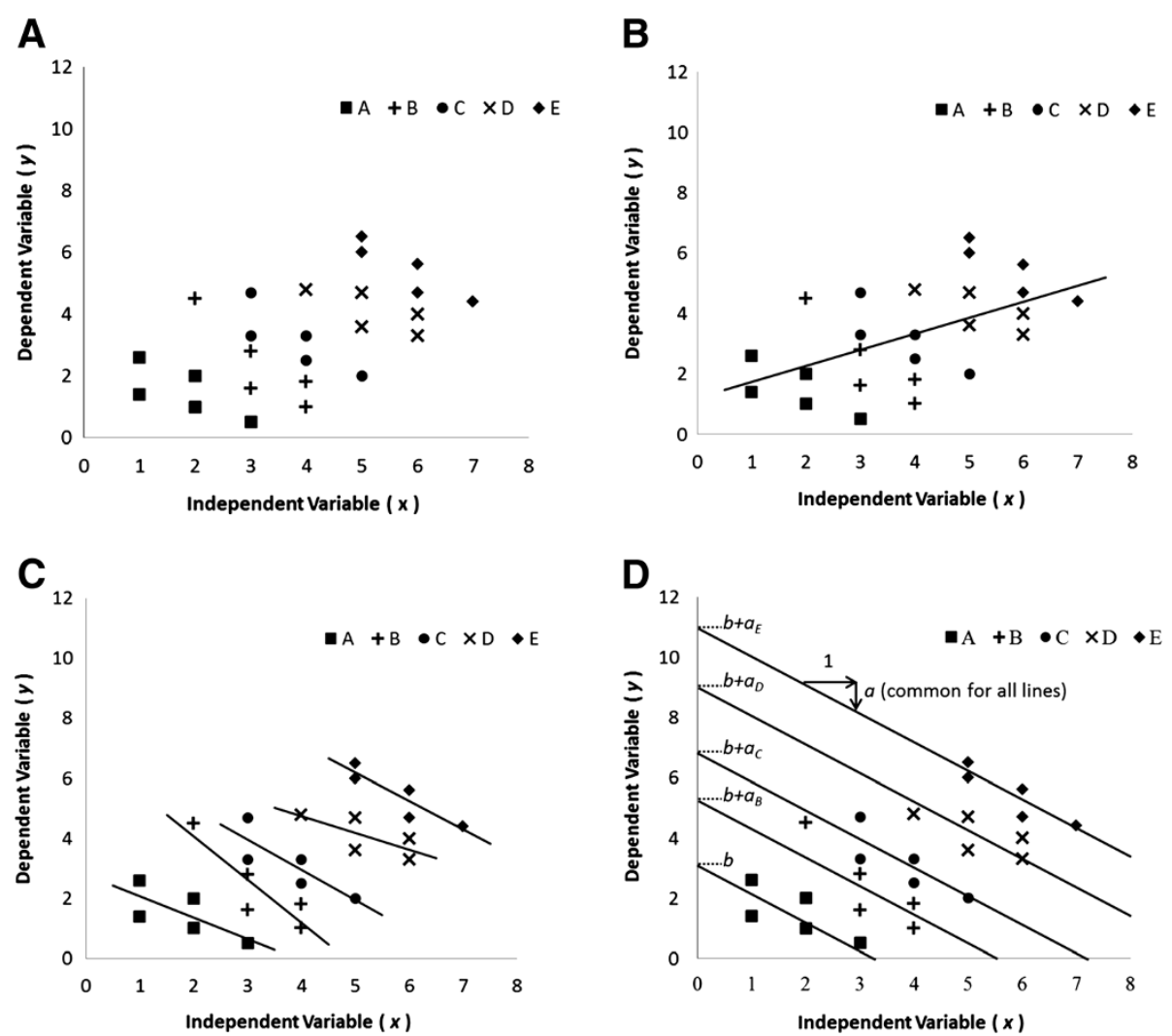

Figure 3 Explanation of correlation calculations. (A) Scatter plot of sample data consisting of five participants. (B) Falsely detected positive correlation between the independent variable and the dependent variable. (C) Intra-individual correlation for each participant. (D) Determining intra-individual correlation using multiple regression analysis. 
subjective impression scores of 'like-dislike' and room air temperature during each session as independent variables.

These statistical analyses were conducted using statistical software (R 2.12.1, R Development Core Team, Vienna, Austria). All tests were two-sided and $P<0.05$ was regarded as statistically significant. When $P<0.1$ this was also taken into consideration as mentioned in the Discussion.

\section{Results}

Participants' impression scores for the videos and thermal sensation scores are shown in Figure 4. The Friedman test revealed statistically significant differences among the videos $(P<0.01)$, meaning that the images used could successfully invoke different levels of hot or cold impressions. All cardiovascular indices for the videos are shown in Figure 5. In $\Delta \mathrm{CO}$, there were significant differences among videos (Friedman test, $P<0.05$ ). In
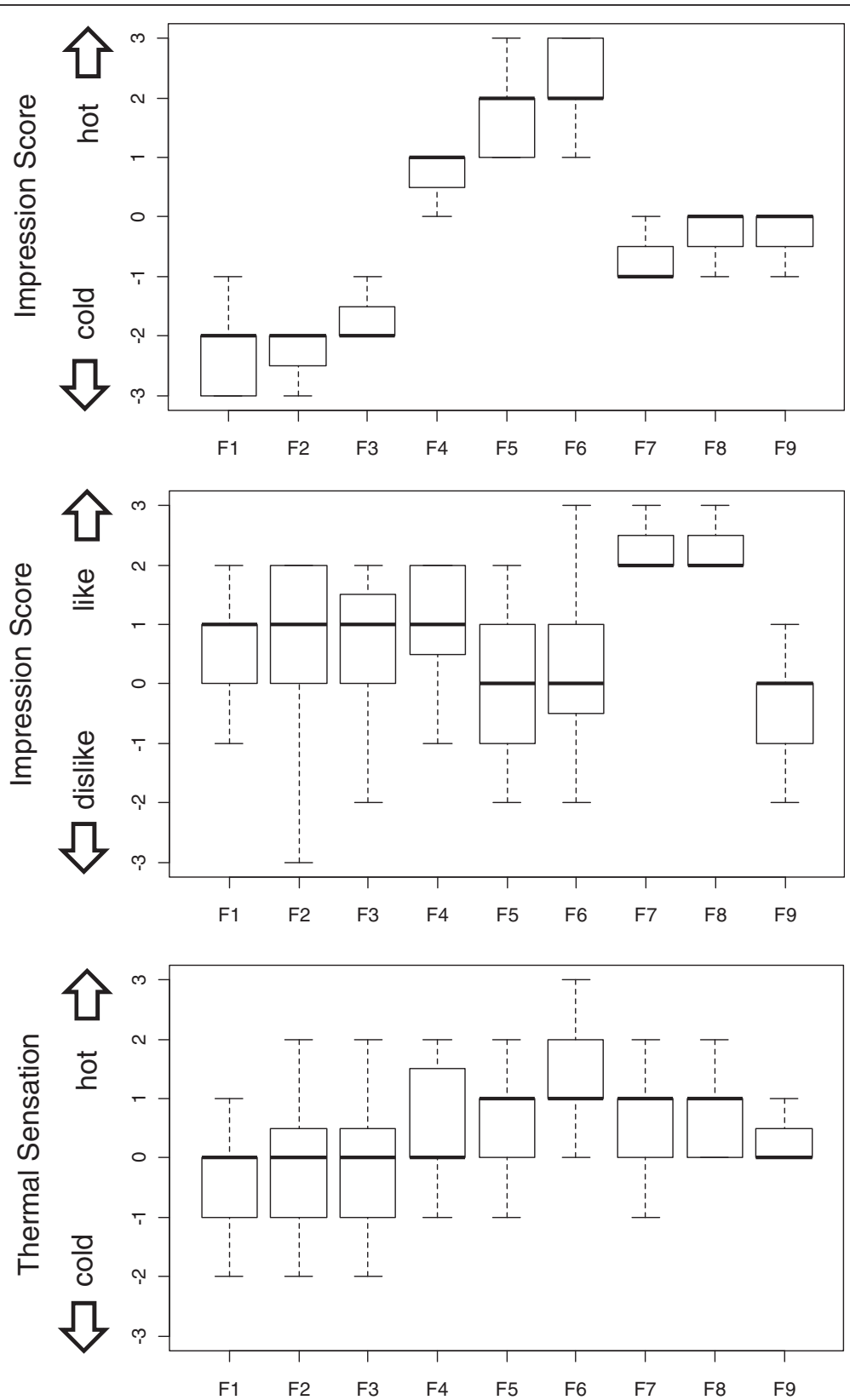

Figure 4 Box and whisker plot of subjective responses. The bars in the plot signify the minimum and maximum values excluding extreme outliers. The top and bottom edges of the boxes indicate the 75th percentile and 25 th percentile values, respectively. The bold lines on the boxes are the median. In the 'hot-cold' impression score, significant differences were found among the values (Friedman test, $P<0.01$ ). 

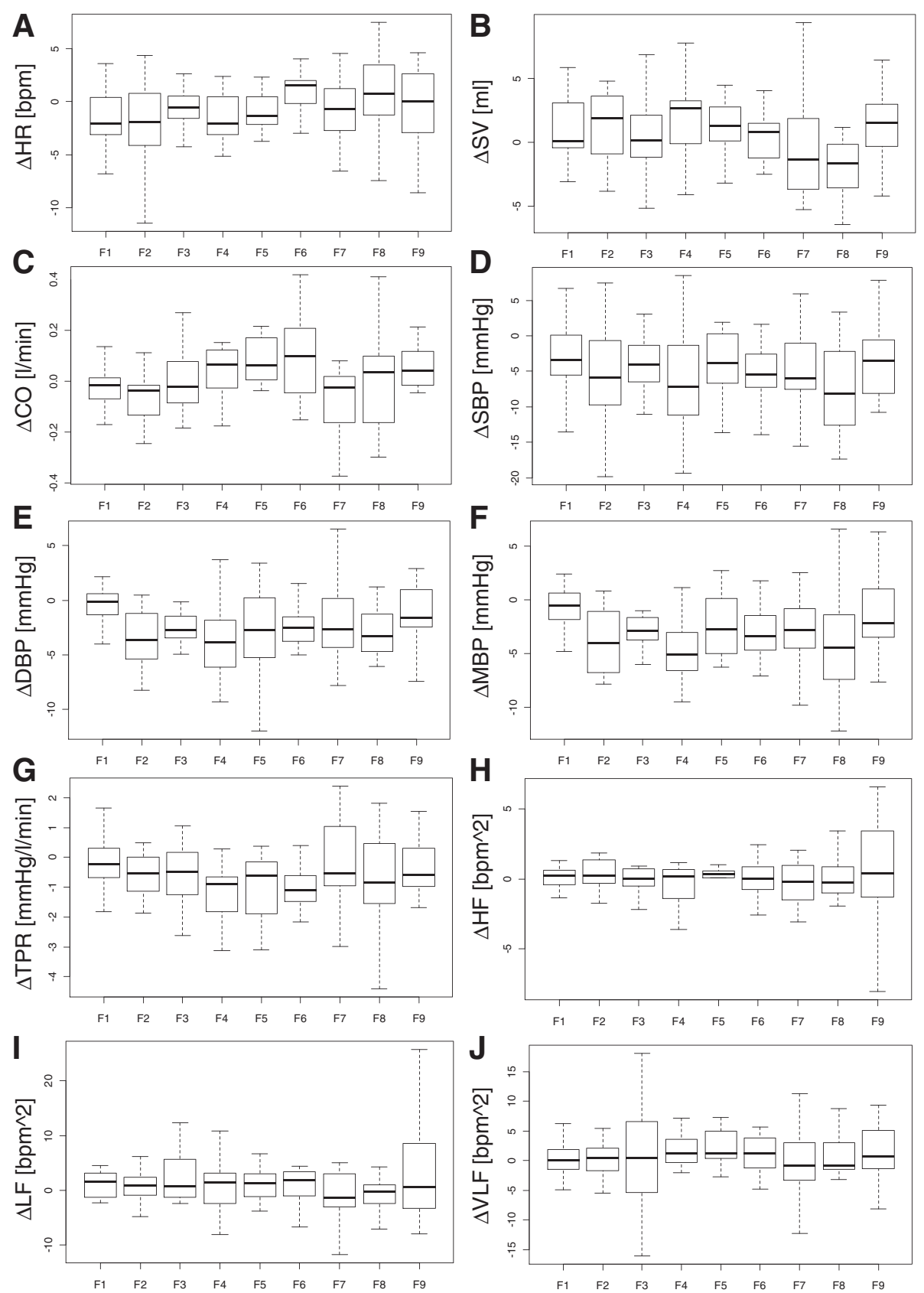

Figure 5 Box and whisker plot of cardiovascular indices. Cardiovascular indices for the videos. Changes from baseline: $(\mathbf{A}) \Delta H R$, heart rate; (B) $\Delta S V$, stroke volume; (C) $\Delta C O$, cardiac output; (D) $\triangle S B P$, systolic blood pressure; (E) $\Delta \mathrm{DBP}$, diastolic blood pressure; (F) $\Delta \mathrm{MBP}$, mean blood pressure; (G) $\triangle T P R$, total peripheral resistance; (H) $\Delta H F$, high frequency HRV; (I) $\Delta L F$, low frequency HRV; and (J) $\Delta V L F$, very low frequency of HRV. The meanings of the bars, edges, and bold lines are the same as in Figure 4. In $\Delta \mathrm{CO}$, significant differences were found among the values (Friedman test, $P<0.05)$. HRV, heart rate variability.

other cardiovascular indices, the results of Friedman tests did not reach significant level.

Table 1 shows the overall results of the correlation analysis. There was a significant positive correlation between 'hot-cold' impression score and $\triangle \mathrm{HR}(\mathrm{r}=0.190$, $P<0.05), \Delta \mathrm{CO}(\mathrm{r}=0.229, P<0.05)$, and $\Delta \operatorname{VLF}(\mathrm{r}=0.190$, $P<0.05)$. Also, a significant negative correlation between the impression score and $\triangle \mathrm{TPR}(\mathrm{r}=0.247, P<0.05)$ was observed. In addition, although it did not reach the level of significance, there was a tendency towards a correlation between the 'hot-cold' impression score and $\triangle \mathrm{DBP}$ $(\mathrm{r}=0.157, P<0.1)$ and $\triangle \mathrm{MBP}(\mathrm{r}=0.163, P<0.1)$. There were no significant correlations between 'hot-cold' impression scores and $\Delta \mathrm{SV}(\mathrm{r}=0.008, P=0.934), \Delta \mathrm{SBP}(\mathrm{r}=0.099$, 
Table 1 Overall results of correlation analysis: impression scores of 'hot-cold'

\begin{tabular}{lccc}
\hline Indices & $\begin{array}{c}\text { Intra-individual } \\
\text { correlation } \\
\text { coefficients }(\boldsymbol{r})\end{array}$ & $\begin{array}{c}\boldsymbol{P} \text { value for null } \\
\text { hypothesis is that } \\
\text { common coefficient } \boldsymbol{a}=\mathbf{0}\end{array}$ & Significance \\
\hline$\Delta \mathrm{HR}$ & +0.190 & 0.037 & $* *$ \\
$\Delta \mathrm{SV}$ & +0.008 & 0.934 & $* *$ \\
$\Delta \mathrm{CO}$ & +0.229 & 0.012 & $*$ \\
$\Delta \mathrm{SBP}$ & -0.099 & 0.282 & $*$ \\
$\Delta \mathrm{DBP}$ & -0.157 & 0.085 & $*$ \\
$\Delta \mathrm{MBP}$ & -0.163 & 0.074 & $*$ \\
$\Delta \mathrm{TPR}$ & -0.247 & 0.006 & $*$ \\
$\Delta \mathrm{HF}$ & +0.111 & 0.224 & $*$ \\
$\Delta \mathrm{LF}$ & +0.071 & 0.437 & $*$ \\
$\Delta \mathrm{VLF}$ & +0.190 & 0.036 & $* 0.001$ \\
Thermal & +0.439 & $<0.000$ & \\
sensation & & & $*$ \\
\hline
\end{tabular}

Correlation coefficients between each index and 'hot-cold' impression scores. Positive correlations mean the hotter the impressions, the greater the indices. ${ }^{*} P<0.1 ;{ }^{* *} P<0.05 ;{ }^{* * *} P<0.01$. The $P$ values are for the common coefficient $a$, not the results of multiple regression analysis. The raw results of multiple regression analyses are shown in Additional file 2. Changes from baseline: $\Delta \mathrm{HR}$, heart rate; $\triangle \mathrm{SV}$, stroke volume; $\triangle \mathrm{CO}$, cardiac output; $\triangle \mathrm{SBP}$, systolic blood pressure; $\triangle \mathrm{DBP}$, diastolic blood pressure; $\triangle \mathrm{MBP}$, mean blood pressure; $\triangle \mathrm{TPR}$, total peripheral resistance; $\Delta H F$, high frequency HRV; $\Delta L F$, low frequency HRV; and $\triangle V L F$, very low frequency of HRV. HRV, heart rate variability.

$P=0.282), \Delta \mathrm{LF}(\mathrm{r}=0.071, P=0.437)$, or $\Delta \mathrm{HF}(\mathrm{r}=0.037$, $P=0.691)$. Here, a positive correlation means the impression is hotter and the physiological index is greater; a negative correlation means the impression is colder and the physiological index is greater. Not surprisingly, there was also a significant positive correlation between the 'hot-cold' impression score and thermal sensation $(\mathrm{r}=0.439, P<0.01)$. The raw results of multiple regression analyses are shown in Additional file 2, and the scatter plots of $\triangle \mathrm{HR}, \triangle \mathrm{CO}$, and $\triangle \mathrm{TPR}$ are shown in Figure 6.

The results of correlation between the cardiovascular indices and possible confounding factors are shown in Table 2 and Table 3. As shown in Table 2, there were not any significant correlations between the cardiovascular indices and the impression score of 'like-dislike'. On the other hand, a significant positive correlation between room air temperature and $\Delta S V(r=0.179, P<0.05)$ was observed. There was also a tendency towards a correlation between $\triangle \mathrm{LF}$ and room air temperature $(\mathrm{r}=0.159$, $P<0.1)$. Room air temperature was significantly correlated with thermal sensation $(\mathrm{r}=0.218, P<0.05)$.

\section{Discussion}

The results show that several cardiovascular indices significantly correlated with the impression score. However, do they resemble the physiological response to actual hot or cold environments? Significant correlations were

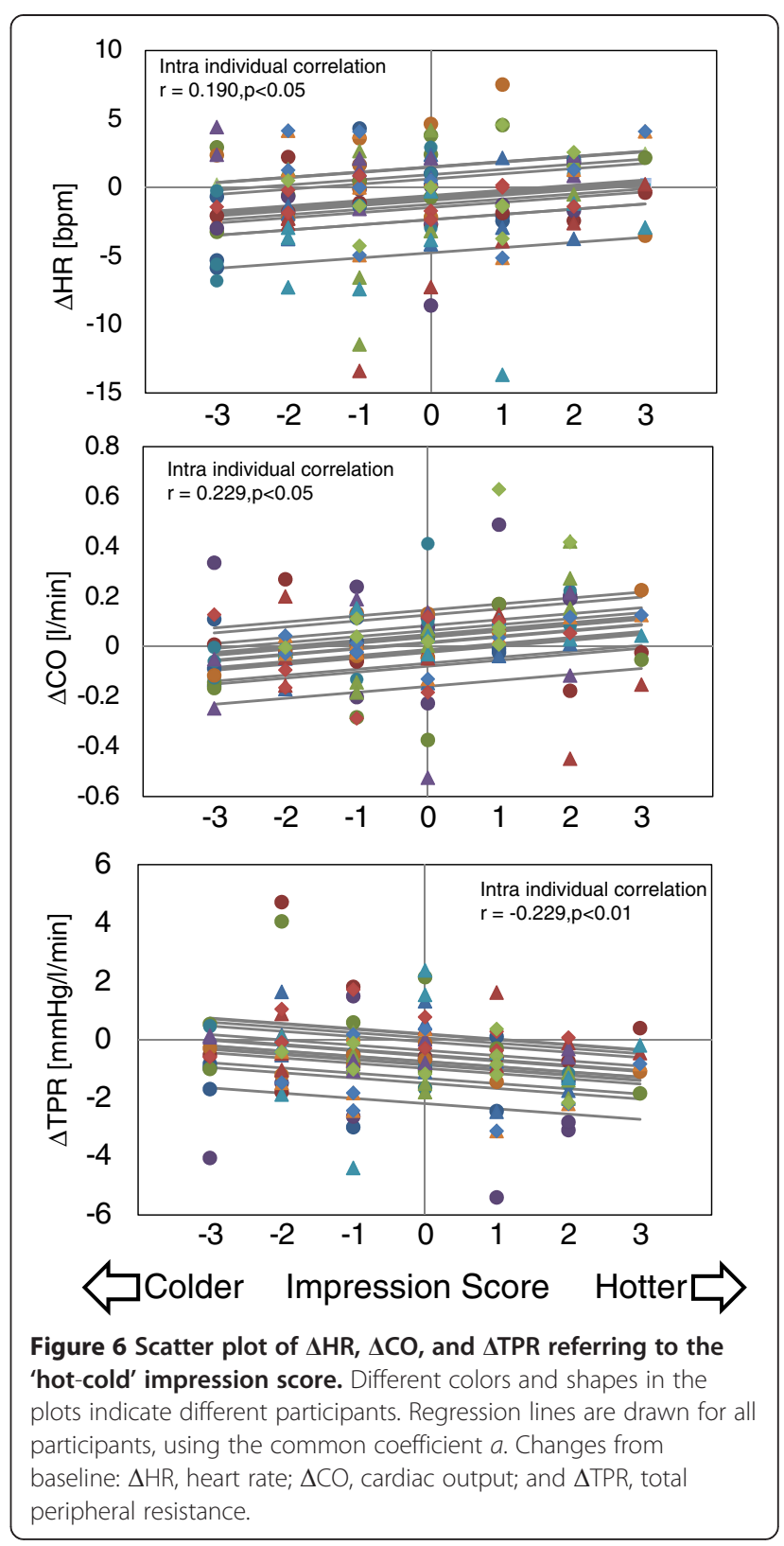

observed in $\triangle \mathrm{HR}$ (positive), $\triangle \mathrm{CO}$ (positive), $\triangle \mathrm{TPR}$ (negative), and $\triangle \mathrm{VLF}$ (positive). The correlation of $\triangle \mathrm{DBP}$ (negative) and $\triangle \mathrm{MBP}$ (negative) also tended towards significance. When we compare each index with that of the actual thermal environment, we find that, in general, when the environment is hot, cardiac output increases, total peripheral resistance decreases, heart rate increases, and blood pressure decreases, while in cold environments the reverse is true [2-4]. Therefore, these five responses $(\Delta H R$, $\triangle \mathrm{CO}, \triangle \mathrm{TPR}, \triangle \mathrm{DBP}$, and $\triangle \mathrm{DBP})$ are similar to those found in actual thermal environments.

On the other hand, the result of $\triangle$ VLF is not so simple. The very low frequency component of HRV is known to 
Table 2 Results of correlation analysis: impression scores of 'like-dislike'

\begin{tabular}{|c|c|c|c|}
\hline Indices & $\begin{array}{l}\text { Intra-individual } \\
\text { correlation } \\
\text { coefficients }(r)\end{array}$ & $\begin{array}{c}P \text { value for null } \\
\text { hypothesis is that } \\
\text { common coefficient } a=0\end{array}$ & Significance \\
\hline$\Delta \mathrm{HR}$ & +0.054 & 0.560 & \\
\hline$\Delta S V$ & -0.090 & 0.328 & \\
\hline$\Delta C O$ & +0.137 & 0.133 & \\
\hline$\triangle \mathrm{SBP}$ & -0.115 & 0.211 & \\
\hline$\triangle \mathrm{DBP}$ & -0.105 & 0.253 & \\
\hline$\triangle \mathrm{MBP}$ & -0.131 & 0.153 & \\
\hline$\triangle T P R$ & -0.027 & 0.767 & \\
\hline$\Delta \mathrm{HF}$ & -0.037 & 0.689 & \\
\hline$\Delta \mathrm{LF}$ & -0.079 & 0.392 & \\
\hline$\Delta \mathrm{VLF}$ & -0.069 & 0.451 & \\
\hline $\begin{array}{l}\text { Thermal } \\
\text { sensation }\end{array}$ & -0.067 & 0.465 & \\
\hline
\end{tabular}

Correlation coefficients between each index and 'like-dislike' impression scores. Positive correlations mean the more pleasant (like) the impressions, the greater the indices. No significant correlations were observed. Changes from baseline: $\Delta \mathrm{HR}$, heart rate; $\Delta \mathrm{SV}$, stroke volume; $\Delta C \mathrm{CO}$, cardiac output; $\triangle \mathrm{SBP}$, systolic blood pressure; $\triangle \mathrm{DBP}$, diastolic blood pressure; $\triangle \mathrm{MBP}$, mean blood pressure; $\triangle T P R$, total peripheral resistance; $\triangle H F$, high frequency $H R V ; \Delta L F$, low frequency $H R V$; and $\triangle V L F$, very low frequency of HRV. HRV, heart rate variability.

increase in both hot and cold environments, but the increase in cold environments is greater [13]. In other words, it shows a mirrored J-shaped response. Therefore, the positive correlation between $\triangle \mathrm{VLF}$ and the impression score does not simply correspond to the response seen in

Table 3 Results of correlation analysis: room air temperature

\begin{tabular}{lccc}
\hline Indices & $\begin{array}{c}\text { Intra-individual } \\
\text { correlation } \\
\text { coefficients }(\mathbf{r})\end{array}$ & $\begin{array}{c}\boldsymbol{P} \text { value for null } \\
\text { hypothesis is that } \\
\text { common coefficient } \boldsymbol{a}=\mathbf{0}\end{array}$ & Significance \\
\hline$\Delta \mathrm{HR}$ & -0.149 & 0.103 \\
$\Delta \mathrm{SV}$ & +0.179 & 0.049 \\
$\Delta \mathrm{CO}$ & +0.131 & 0.152 \\
$\Delta \mathrm{SBP}$ & +0.046 & 0.614 \\
$\Delta \mathrm{DBP}$ & +0.016 & 0.860 \\
$\Delta \mathrm{MBP}$ & +0.032 & 0.725 \\
$\Delta \mathrm{TPR}$ & -0.052 & 0.571 \\
$\Delta \mathrm{HF}$ & +0.089 & 0.331 \\
$\Delta \mathrm{LF}$ & +0.159 & 0.082 \\
$\Delta \mathrm{VLF}$ & -0.082 & 0.373 \\
Thermal & +0.218 & 0.016 \\
sensation & & $*$ \\
\hline Coretion & & $*$
\end{tabular}

Correlation coefficients between each index and room air temperature. Positive correlations mean the warmer the temperature, the greater the indices. ${ }^{*} P<0.1 ;{ }^{* *} P<0.05$. Changes from baseline: $\Delta \mathrm{HR}$, heart rate; $\Delta S V$, stroke volume; $\triangle \mathrm{CO}$, cardiac output; $\triangle \mathrm{SBP}$, systolic blood pressure; $\triangle \mathrm{DBP}$, diastolic blood pressure; $\triangle M B P$, mean blood pressure; $\triangle T P R$, total peripheral resistance; $\Delta \mathrm{HF}$, high frequency $H R V ; \Delta L F$, low frequency $H R V$; and $\Delta V L F$, very low frequency of HRV. HRV, heart rate variability. the actual thermal environment. However, if we regard this result as the right half of the mirrored J shape, we can conclude that $\triangle \mathrm{VLF}$ is similar to the response in the actual environment. However, since we cannot identify which area of the mirrored J shape appears in this experiment, the above interpretation can be misleading. In addition, a very low frequency of HRV is, in fact, very slow fluctuation. Therefore, it is possible that heart rate fluctuations correspond to the variation among the videos. If so, $\triangle \mathrm{VLF}$ should not be considered as either a spontaneous oscillation of heart rate or as the index of renin-angiotensin axis-related vasomotor activity [14]. We cannot conclude whether there are similarities to the actual environment based on $\triangle \mathrm{VLF}$.

From the viewpoint of thermoregulation, cardiac output and total peripheral resistance are the important indices because overall thermal energy flow between the body and the environment is essentially determined by these two parameters. In our results, among the other cardiovascular indices, $\triangle \mathrm{CO}$ and $\triangle \mathrm{TPR}$ did resemble the actual thermoregulatory responses according to the two highest correlation coefficients. This means that the responses in this study may actually contribute to the thermal energy flow to a certain degree (unfortunately, we cannot quantify this from the present data). There are two possible ways to increase cardiac output. One is to increase heart rate and the other is to increase stroke volume. In our results, $\Delta \mathrm{HR}$ correlated significantly with the impressions, while $\Delta \mathrm{SV}$ did not. This implies that the change in $\triangle C O$ depends on the change in $\triangle \mathrm{HR}$ rather than on $\Delta \mathrm{SV}$. Notably, the change in cardiac output depends on the heart rate in actual thermoregulatory responses as well [2-4].

Regarding the relationship among cardiac output, total peripheral resistance, and blood pressure, in thermoregulatory responses the change in total peripheral resistance tends to be greater than that of cardiac output, and blood pressure decreases in hot environments and increases in cold environments [2-4]. This tendency was observed in the present study (that is, the hotter the impression, the lower the blood pressure).

As mentioned above, six cardiovascular indices showed a significant or nearly significant correlation to the impression score, and at least five of these six were in concordance with the actual thermal environment. Such hemodynamic relationships may be evidence that regional sympathetic nerve activity [15], which is specific in thermoregulatory responses, was activated in this experiment. Therefore, we can say that apparent cardiovascular responses are similar to those corresponding to actual thermal environments.

On the other hand, the effect sizes observed in this experiment are not large and there are some possible confounding factors as mentioned in the Methods. It is well 
known that pleasant or unpleasant feelings can induce cardiovascular responses [16]. However there were not any significant correlations with 'like-dislike' impression scores, indicating that pleasantness or unpleasantness did not affect this experiment. There were significant or nearly significant correlations between room air temperature and $\triangle \mathrm{SV}$ and $\triangle \mathrm{LF}$, but these two indices did not correlate with the 'hot-cold' impression score. While room air temperature significantly correlated with thermal sensation, the effect size is much smaller than that of the 'hot-cold' impression score. Therefore, even considering such confounding factors, thermoregulatory-like cardiovascular responses observed in this experiment seem to be attributed to mainly 'hot-cold' visual information, but not conclusive.

Although this result is quite interesting, is such a response possible from the viewpoint of physiological mechanisms? One possible explanation of this phenomenon is classical conditioning [17] (so-called Pavlovian conditioning). In a study conducted by Kojo [18], participants immersed a hand in hot or cold water while hearing the sound of streaming water. Afterwards, when they heard the sound of streaming water, their skin temperature changed. This can be interpreted as a typical example that classical conditioning can also affect human temperature regulation.

In our study, however, all participants lived in Japan and most of them had never seen the Arctic Ocean, erupting volcanoes, tropical forests, or large deserts in person. Therefore, they had 'known' but had not 'experienced' the thermal conditions in the videos before the experiment. This may seem somewhat puzzling, but past studies on classical conditioning and/or brain anatomy may be able to explain this paradox.

Firstly, in classic human conditioning responses, it is known that imagery of unconditioned stimuli is more important than conditional stimuli per se [19]. Therefore, if mental imagery of hot or cold thermal environments were evoked by the video films, such unconditioned responses (thermoregulatory-like cardiovascular responses) could occur. Secondly, a recent brain imaging study revealed that both remembering and knowing share some common brain areas [20]. Taking these facts together, we believe that it is possible for visual information to induce thermoregulatory-like physiological responses. The question is whether these thermoregulatory-like cardiovascular responses 'intend' to regulate body temperature. A connectivity study indicated that the preoptic area of the hypothalamus, which is the central component of body temperature regulation, has extrinsic connections from the cerebral cortices [21]. If this pathway were involved in these responses, we could say that it is a thermoregulatory physiological response, but we cannot draw this conclusion from the present study alone.
A major weakness of this study is that we did not measure the heat radiation (mean skin temperature), heat production $\left(\mathrm{O}_{2}\right.$ uptake), core body temperature (rectal temperature), and so on. Additional measurement cables induced great ham noises particularly on the impedance cardiograph because of the grounding condition of the laboratory. In addition, we did not want the participants to notice that we focused on the thermoregulatory responses. Small effect sizes (correlation coefficients are not large) should also be considered regardless of statistical significance. Measuring heat radiation, heat production, and core body temperature directly will uncover whether video images affect actual thermoregulation and would be an interesting future study, but more elaborate experimental design is needed. In addition this experiment was conducted in autumn in Japan for Japanese participants. It is known that thermoregulatory responses are different among seasons [22] and among ethnic groups [23]. Investigating such variations is also important from the viewpoint of physiological anthropology. These themes will be important for future studies too.

The main purpose of the human body temperature regulation system is to keep the core body temperature constant. Consequently, it is not useful for it to have sensitivity to anything that does not convey thermal energy (for example, the content of videos). Our results suggest that the human thermoregulatory system can 'wrongly' respond to such visual information, at least superficially. From the viewpoint of evolution, such physiological mechanisms were not maladaptive to human ancestors, as they rarely encountered situations in which visual information did not match actual thermal stimuli. However, today's rapid increase in media technology and artificial environments may uncover this inherent defect of the thermoregulatory system, and may have negative effects on human homeostasis. To confirm such possibilities, further study is needed.

\section{Conclusions}

We found that when participants viewed videos evoking hot or cold impressions in a thermally constant environment, their cardiovascular indices changed as if the actual thermal environment had varied corresponding to the impressions. While it is not clear how such responses were evoked, it is possible that such a propensity could result in the disturbance of homeostasis when the thermal environment and visual information are dissonant. Considering the rapid increase in media technology, it is quite easy to create dissonant artificial environments in today's world, thus great care should be paid when designing such environments, and more study is needed. 


\section{Additional files}

Additional file 1: The explanation that the multiple regression analysis can discriminate whether the tendency is common among participants or not.

Additional file 2: Table S2. Results of multiple regression analysis using dummy variables.

\section{Abbreviations}

ECG: Electrocardiography; HRV: Heart rate variability.

\section{Competing interests}

The authors declare that they have no financial competing interests on disclosing this article.

\section{Authors' contributions}

JT conducted the experiment, analyzed the results, and wrote the manuscript. TN participated in the discussion and preparation of the manuscript. SW planned the experiment, participated in the discussion, and authorized the total study. All authors read and approved the final manuscript.

\section{Acknowledgements}

This study was partly funded by the Japan Society for the Promotion of Science (grant no: 24657175).

\section{Author details}

${ }^{1}$ Graduate School of Integrated Frontier Science, Kyushu University, 4-9-1, Shiobaru, Minami-Ku, Fukuoka, Japan. 'Department of Public Health, Nagasaki University Graduate School of Biomedical Sciences, 1-12-4, Sakamoto, Nagasaki, Japan. ${ }^{3}$ Faculty of Design, Kyushu University, 4-9-1, Shiobaru, Minami-KuFukuoka, Japan.

Received: 18 May 2013 Accepted: 27 November 2013

Published: 28 December 2013

\section{References}

1. Boulant JA: Hypothalamic neurons. Mechanisms of sensitivity to temperature. Ann N Y Acad Sci 1998, 856:108-115.

2. Rowell LB, Brengelmann GL, Murray JA: Cardiovascular responses to sustained high skin temperature in resting man. J Appl Physiol 1969, 27:673-680.

3. Crandall CG, González-Alonso J: Cardiovascular function in the heat-stressed human. Acta Physiol (Oxf) 2010, 199:407-423.

4. Kinugasa $\mathrm{H}$, Hirayanagi K: Effects of skin surface cooling and heating on autonomic nervous activity and baroreflex sensitivity in humans. Exp Physiol 1999, 84:369-377.

5. Kanosue K, Crawshaw LI, Nagashima K, Yoda T: Concepts to utilize in describing thermoregulation and neurophysiological evidence for how the system works. Eur J Appl Physiol 2010, 109:5-11.

6. Fraden J: Handbook of Modern Sensors: Physics, Designs, and Applications. Berlin: Springer; 2010

7. Dawkins R: The Blind Watchmaker. London: Penguin Group; 1986.

8. Yampolsky LY, Kondrashov FA, Kondrashov AS: Distribution of the strength of selection against amino acid replacements in human proteins. Hum Mol Genet 2005, 14:3191-3201.

9. Marshall SJ, Gorely T, Biddle SJ: A descriptive epidemiology of screen-based media use in youth: a review and critique. J Adolesc 2006, 29:333-349.

10. Kubicek WG, Karnegis JN, Patterson RP, Witsoe DA, Mattson RH: Development and evaluation of an impedance cardiac output system. Aerosp Med 1966, 37:1208-1212.

11. Barros AK, Yoshizawa M, Yasuda Y: Filtering noncorrelated noise in impedance cardiography. IEEE Trans Biomed Eng 1995, 42:324-327.

12. Bland JM, Altman DG: Calculating correlation coefficients with repeated observations: Part 1-Correlation within subjects. BMJ 1995, 310:446.

13. Sollers JJ 3rd, Sanford TA, Nabors-Oberg R, Anderson CA, Thayer JF: Examining changes in $\mathrm{HRV}$ in response to varying ambient temperature. IEEE Eng Med Biol Mag 2002, 21:30-34.
14. Fleisher LA, Frank SM, Sessler DI, Cheng C, Matsukawa T, Vannier CA Thermoregulation and heart rate variability. Clin Sci (Lond) 1996, 90:97-103.

15. Walther $\mathrm{OE}$, Iriki $\mathrm{M}$, Simon E: Antagonistic changes of blood flow and sympathetic activity in different vascular beds following central thermal stimulation. II. Cutaneous and visceral sympathetic activity during spinal cord heating and cooling in anesthetized rabbits and cats. Pflugers Arch 1970, 319:162-184.

16. Ekman P, Levenson RW, Friesen W: Autonomic nervous system activity distinguishes among emotions. Science 1983, 221:1208-1210.

17. Nolen-Hoeksema S, Fredrickson BL, Loftus GR, Wagenaar WA: Learning and conditioning. In Atkinson \& Hilgard's Introduction to Psychology. 15th edition. Andover: Cengage Learning; 2009:236-269.

18. Kojo I: The effects of mental imagery on skin temperature and skin temperature sensation. Scand J Psychol 1985, 26:314-320.

19. Dadds MR, Bovbjerg DH, Redd WH, Cutmore TR: Imagery in human classical conditioning. Psychol Bull 1997, 122:89-103.

20. Wheeler ME, Buckner RL: Functional-anatomic correlates of remembering and knowing. Neuroimage 2004, 21:1337-1349.

21. Lemaire JJ, Frew AJ, MCArthur D, Gorgulho AA, Alger JR, Salomon N, Chen C, Behnke EJ, De Salles AA: White matter connectivity of human hypothalamus. Brain Res 2011, 1371:43-64

22. Nishimura $T$, Motoi M, Niri $Y$, Hoshi $Y$, Kondo R, Watanuki S: Relationship between seasonal cold acclimatization and mtDNA haplogroup in Japanese. J Physiol Anthropol. 2012, 31:22.

23. Wijayanto $T$, Toramoto $S$, Wakabayashi H, Tochihara Y: Effects of duration of stay in temperate area on thermoregulatory responses to passive heat exposure in tropical south-east Asian males residing in Japan. J Physiol Anthropol 2012, 31:25.

\section{doi:10.1186/1880-6805-32-26}

Cite this article as: Takakura et al:: Visual information without therma energy may induce thermoregulatory-like cardiovascular responses. Journal of Physiological Anthropology 2013 32:26.

\section{Submit your next manuscript to BioMed Central and take full advantage of:}

- Convenient online submission

- Thorough peer review

- No space constraints or color figure charges

- Immediate publication on acceptance

- Inclusion in PubMed, CAS, Scopus and Google Scholar

- Research which is freely available for redistribution 